\title{
Instability of transonic flow past flattened airfoils
}

Communication

\author{
Alexander Kuzmin ${ }^{1 *}$ \\ 1 St. Petersburg State University, Department of Mathematics and Mechanics \\ 28 University Avenue, 198504 St Petersburg, Russia
}

Received 15 June 2013; accepted 28 August 2013

Abstract: Transonic flow past a Whitcomb airfoil and two modifications of it at Reynolds numbers of the order of ten millions is studied. The numerical modeling is based on the system of Reynolds-averaged Navier-Stokes equations. The flow simulations show that variations of the lift coefficient versus the angle of attack become more abrupt with decreasing curvature of the airfoil in the midchord region. This is caused by an instability of closely spaced local supersonic regions on the upper surface of the airfoil.

Keywords: Local supersonic regions $\cdot$ Shock waves $・$ Interaction $・$ Instability

(c) Versita sp. z o.o.

\section{Introduction}

In the last two decades, a number of numerical studies have shown the instability of shock waves on airfoils comprising a flat or nearly flat arc [1-4]. The instability is associated with an interaction of local supersonic regions that arise near endpoints of the arc and expand with increasing free-stream Mach number $M_{\infty}$. The expansion followed by a coalescence of the supersonic regions causes abrupt changes of shock wave positions and jumps of aerodynamic coefficients. An opposite phenomenon is a rupture of the supersonic region on the flattened arc of the airfoil with decreasing $M_{\infty}$. It again causes abrupt changes of the aerodynamic coefficients. The coalescence and rupture of supersonic regions were scrutinized for several symmetric profiles $[4,5]$ and the asymmetric J78 airfoil [4]. Recently the instability was explored for the asymmetric Apex 16 airfoil [6] and a Whitcomb air-

*E-mail: alexander.kuzmin@pobox.spbu.ru

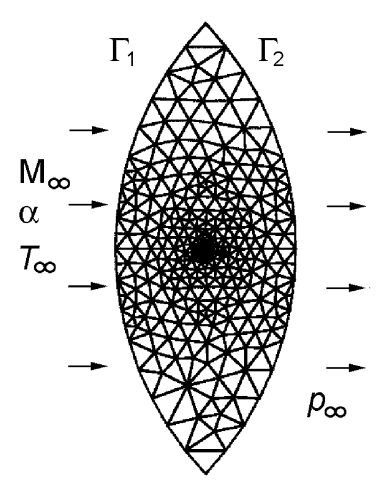

Figure 1. Sketch of the computational domain and mesh.

foil with a deflected aileron (A. Kuzmin and A. Ryabinin, http://www.iccfd.org/iccfd7 ). In this paper, we study two more modifications of the Whitcomb airfoil and confirm the crucial role of the existence of a flattened arc for the transonic flow instability.

In practice, airfoils with a small local curvature in the midchord region may arise as a solution of a design opti- 
mization problem if one employs an unsophisticated cost function, e.g., a smooth pressure or velocity distribution on the airfoil $[1,7,8]$. Also the small local curvature may occur in a transonic flow control by airfoil modifications with a small bump in the shock foot area $[9,10]$. Though the optimized/adapted airfoils demonstrate higher aerodynamic performance at design free-stream conditions, they typically exhibit poor characteristics at off-design ones. That is why, in late 1990s, some researchers suggested advanced optimization methods that mitigate the decrease of the airfoil performance at off-design conditions [11, 12]. However, the underlying causes of the decrease have not been unveiled.

\section{Problem Formulation and a Nu- merical Method}

We consider a family of airfoils given by the expression as follows:

$$
y(x)=\left(1-p x+p x^{2}\right) y_{\text {whit }}(x), \quad 0 \leq x \leq 1,
$$

where $(x, y)$ are non-dimensional Cartesian coordinates, $p \geq 0$ is a parameter, and $y_{\text {whit }}(x)$ refers to the upper and lower surfaces of the Whitcomb integral supercritical airfoil (http://www.ae.illinois.edu/m-selig ). Due to the factor $\left(1-p x+p x^{2}\right)$, the thickness and curvature of airfoil (1) in the midchord region decrease with the increase of the parameter $p$.

The airfoil is placed at the center of a lens-shaped computational domain, which is bounded by two circular arcs, $\Gamma_{1}$ and $\Gamma_{2}$, see Fig. 1. The width and height of the domain are 80 and 200, respectively. On the inflow part $\Gamma_{1}$ of the boundary, we prescribe stationary values of the angle of attack $\alpha$, the Mach number $M_{\infty}<1$, and the static temperature $T_{\infty}$. On the outflow part $\Gamma_{2}$ of the boundary, the static pressure $p_{\infty}$ is given. On the airfoil, the no-slip condition and vanishing flux of heat are employed. The specific heat at constant pressure is $1004.4 \mathrm{~J} /(\mathrm{kg} \cdot \mathrm{K})$, while the one at constant volume is $717.3 \mathrm{~J} /(\mathrm{kg} \cdot \mathrm{K})$. We adopted a value of $28.96 \mathrm{~kg} / \mathrm{kmol}$ for the molar mass and used the Sutherland formula for the molecular dynamic viscosity. Initial data were parameters of the uniform free-stream, in which the turbulence level was set to $1 \%$.

Solutions of the RANS equations were obtained with an ANSYS CFX-13 finite-volume solver based on a highresolution scheme by Barth and Jespersen [13]. For the time-stepping we used an implicit second-order accurate backward Euler scheme. Computations were performed primarily on hybrid unstructured meshes of about $5 \times 10^{5}$ grid points, which were clustered in the boundary layer,
Table 1. Free-stream parameters in the case of airfoil's chord length $L_{\text {chord }}=0.5 \mathrm{~m}$.

\begin{tabular}{llll}
\hline $\begin{array}{l}p_{\infty}, \mathrm{N} / \mathrm{m}^{2} \\
\text { static pressure }\end{array}$ & $\begin{array}{c}\rho_{\infty}, \mathrm{kg} / \mathrm{m}^{3} \\
\text { density }\end{array}$ & $\begin{array}{l}T_{\infty}, \mathrm{K} \\
\text { temperature }\end{array}$ & $\begin{array}{l}a_{\infty}, \mathrm{m} / \mathrm{s} \\
\text { sound speed }\end{array}$ \\
\hline 50,000 & 0.6966 & 250 & 317.02 \\
\hline
\end{tabular}

Table 2. Free-stream parameters in the case $L_{\text {chord }}=2.5 \mathrm{~m}$.

\begin{tabular}{llll}
\hline $\begin{array}{l}p_{\infty}, \mathrm{N} / \mathrm{m}^{2} \\
\text { static pressure }\end{array}$ & $\begin{array}{l}\rho_{\infty}, \mathrm{kg} / \mathrm{m}^{3} \\
\text { density }\end{array}$ & $\begin{array}{l}T_{\infty}, \mathrm{K} \\
\text { temperature }\end{array}$ & $\begin{array}{l}a_{\infty}, \mathrm{m} / \mathrm{s} \\
\text { sound speed }\end{array}$ \\
\hline 26,434 & 0.4126 & 223.15 & 299.51 \\
\hline
\end{tabular}

in the wake, and in the vicinities of shock waves. The non-dimensional thickness $y^{+}$of the first mesh layer on the airfoil was chosen less than 1 in order to provide a good resolution of the boundary layer. We normally used the standard $k-\omega$ SST turbulence model, which reasonably predicts flows with boundary layer separations from smooth surfaces. In addition, in a few cases we employed a BSL Reynolds stress turbulence model [14] based on differential transport equations with a low-Reynolds formulation near the solid walls. The first turbulence model is widely used in aerodynamic applications, whereas the second one is more elaborate and promising but needs some improvements with respect to its robustness.

Verification of the solver included a comparison of solutions with available experimental and numerical data for several benchmark transonic problems. In particular, we obtained a good agreement of the lift coefficient $C_{L}(\alpha)$ calculated for a RAE 2822 airfoil at angles of incidence in the range $0 \leq \alpha \leq 3.2 \mathrm{deg}$ with results presented in [15-17].

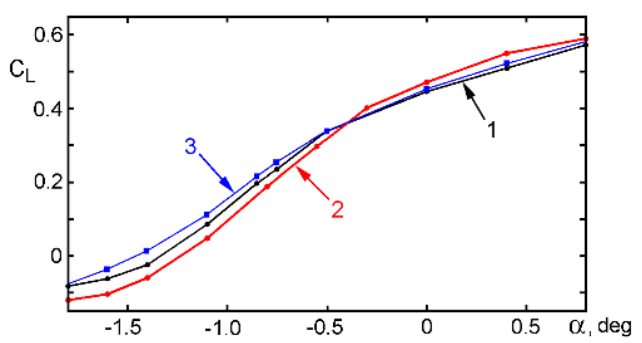

Figure 2. Lift coefficient as a function of the angle of attack for the Whitcomb integral airfoil at $M_{\infty}=0.812$ : plot $1-$ airfoil's chord length $L_{\text {chord }}=0.5 \mathrm{~m}, \quad \operatorname{Re}=4.9 \times 10^{6}$, $k-\omega$ SST turbulence model; plot $2-L_{\text {chord }}=2.5 \mathrm{~m}$, $R e=1.37 \times 10^{7}, k-\omega$ SST model; plot $3-L_{\text {chord }}=0.5$ $\mathrm{m}, \operatorname{Re}=4.9 \times 10^{6}$, BSL Reynolds stress model. 


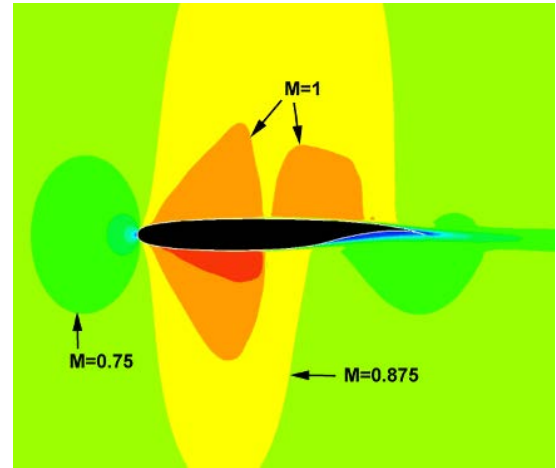

Figure 3. Whitcomb integral airfoil: Mach number contours at $M_{\infty}=$ $0.812, R e=1.37 \times 10^{7}, \alpha=-0.8 \mathrm{deg}, \quad k-\omega \mathrm{SST}$, $L_{\text {chord }}=2.5 \mathrm{~m}$, and free-stream conditions given in Table 2.

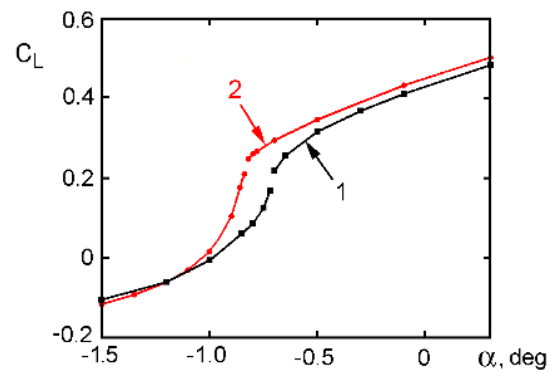

Figure 4. Airfoil (1) with $p=0.5$, the lift coefficient as a function of the angle of attack at $M_{\infty}=0.836, k-\omega$ SST turbulence model: plot $1-L_{\text {chord }}=0.5 \mathrm{~m}, R e=5.0 \times 10^{6} ;$ plot $2-$ $L_{\text {chord }}=2.5 \mathrm{~m}, R e=1.41 \times 10^{7}$.

\section{Results of the Numerical Simula- tion}

The numerical simulation of transonic flow was performed for two sets of free-stream parameters that are pointed out in Tables 1 and 2. The parameters given in Table 2 are respective to the standard atmosphere at a height of $10 \mathrm{~km}$.

For airfoil (1) with $p=0$, i.e. the original $11 \%$-thick Whitcomb airfoil, the obtained plots of lift coefficient $C_{L}$ versus the angle of attack $\alpha$ exhibit noticeable inflections. In particular, Fig. 2 shows plots calculated at the Mach number $M_{\infty}=0.812$. The inflections at $\alpha \approx-0.6 \mathrm{deg}$ can be explained by an interplay of local supersonic regions formed on the upper surface of the airfoil, see Fig. 3.

For $p=0.5$, the thickness of airfoil (1) is reduced to $9.7 \%$. The smaller thickness results in a shift of the transonic regime of interest to higher values of $M_{\infty}$, whereas

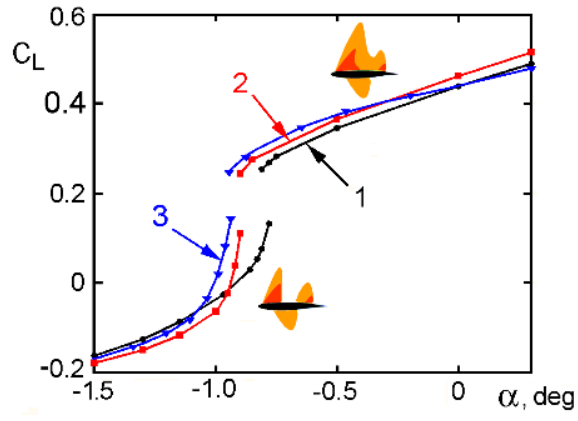

Figure 5. Airfoil (1) with $p=1$, the lift coefficient as a function of the angle of attack at $M_{\infty}=0.85$ : plot $1-L_{\text {chord }}=0.5$ $\mathrm{m}, k-\omega$ SST turbulence model; plot $2-L_{\text {chord }}=2.5 \mathrm{~m}$, $k-\omega$ SST model; plot $3-L_{\text {chord }}=0.5 \mathrm{~m}$, BSL Reynolds stress model.

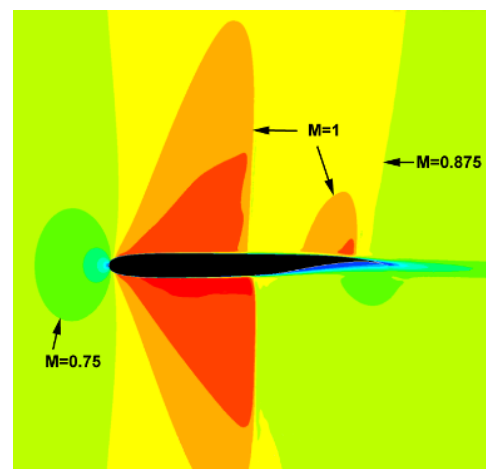

Figure 6. Airfoil (1) with $p=1$ : Mach number contours at $M_{\infty}=$ $0.85, \operatorname{Re}=1.43 \times 10^{7}, \alpha=-0.9 \mathrm{deg}, k-\omega \mathrm{SST}, L_{\text {chord }}=$ $2.5 \mathrm{~m}$.

the smaller curvature of the upper surface enhances the steepness of plots $C_{L}(\alpha)$ at the inflection points. The maximum slope is attained at $M_{\infty} \approx 0.836, \alpha \approx-0.8 \mathrm{deg}$ and it looks like a small discontinuity, see Fig. 4.

For $p=1$, the thickness of airfoil (1) and the curvature of the upper surface in the midchord region are further reduced, so that the surface becomes nearly flat in the interval $0.3<x<0.7$. The flow simulation reveals evident discontinuities in the plots $C_{L}(\alpha)$ at

$$
0.818<M_{\infty}<1
$$

and

$$
0.816<M_{\infty}<1
$$

in the cases pointed out in Tables 1 and 2, respectively. In particular, Fig. 5 displays the obtained lift coefficient at $M_{\infty}=0.85$. The discontinuities are caused by the flow instability associated with a coalescence/rupture of the 


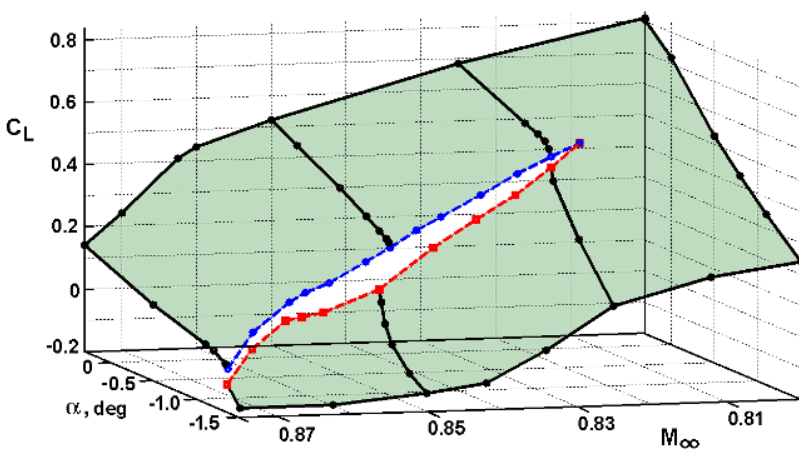

Figure 7. Airfoil (1) with $p=1$ : lift coefficient as a function of the angle of attack and $M_{\infty}$ at $R e \approx 5.1 \times 10^{6}, k-\omega \mathrm{SST}$, $L_{\text {chord }}=0.5 \mathrm{~m}$.

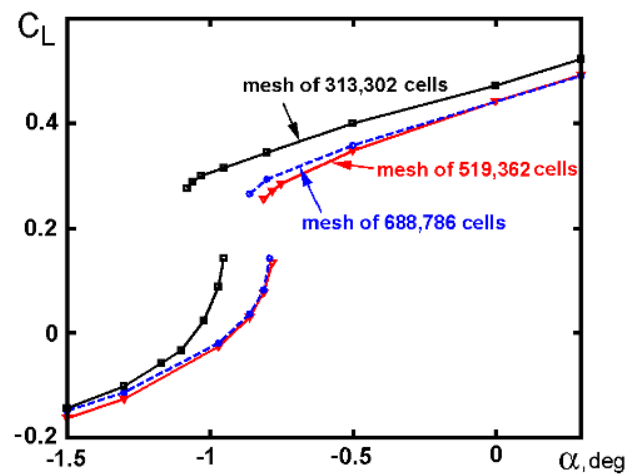

Figure 8. Airfoil (1) with $p=1$ : the lift coefficient as a function of the angle of attack calculated on three different meshes at $M_{\infty}=0.85, k-\omega \mathrm{SST}, L_{\text {chord }}=0.5 \mathrm{~m}, R e=5.1 \times 10^{6}$.

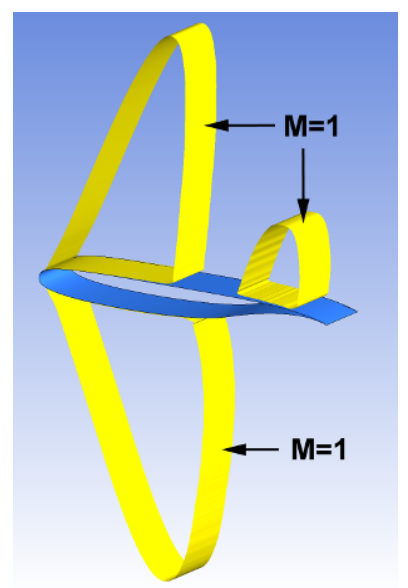

Figure 9. Local supersonic regions on the extruded airfoil (1) configuration at $p=1, M_{\infty}=0.85, \alpha=-0.9 \mathrm{deg}, R e=$ $1.43 \times 10^{7}, k-\omega \mathrm{SST}, L_{\text {chord }}=2.5 \mathrm{~m}, L_{\text {span }}=0.2 L_{\text {chord }}$. supersonic regions on the upper surface of the airfoil, see Fig. 6.

Figure 7 illustrates a dependence of the lift coefficient on two free-stream parameters, $\alpha$ and $M_{\infty}$, for airfoil (1) with $p=1, \quad L_{\text {chord }}=0.5 \mathrm{~m}$. As seen, the surface $C_{L}\left(\alpha, M_{\infty}\right)$ exhibits a slit, whose upper and lower edges correspond to the discontinuities of the plots $C_{L}(\alpha)$ in the range (2) of $M_{\infty}$

For verification of the obtained solutions, we recomputed the case respective to curve 1 in Fig. 5 on three different meshes. A comparison of the plots presented in Fig. 8 shows that the solution obtained on the mesh of 519,362 cells is accurate enough.

In order to examine an influence of 3D phenomena, we performed computations of transonic flow past an unswept wing of $0.2 L_{\text {chord }}$ span with the airfoil (1) at cross sections. A computational mesh was created by the extrusion of $2 \mathrm{D}$ mesh with 30 cells in the spanwise direction. A condition of symmetry [14] was prescribed on the boundaries $z=$ 0 and $z=0.2 L_{\text {chord. }}$. The flow simulation has confirmed the formation of a double supersonic region on the upper surface of the wing (see Fig. 9) and the flow sensitivity to small perturbations of free-stream conditions.

\section{Conclusion}

The numerical simulation showed that by increasing parameter $p$ and, consequently, decreasing the curvature of the airfoil (1) in the midchord region, the maximum slope of the plots $C_{L}(\alpha)$ increases and eventually turns into a discontinuity. This confirms the concept suggested in the previous papers [3] that airfoils comprising a flattened arc admit the flow instability in certain adverse free-stream conditions.

\section{Acknowledgments}

This research was carried out using computational resources provided by the Computational Center of St. Petersburg State University (http://cc.spbu.ru). The work was supported by the Russian Foundation for Basic Research under a grant no. 13-08-00288.

\section{References}

[1] Jameson, A., Airfoils admitting non-unique solutions of the Euler equations, AIAA Paper, 1991, 91-1625, $1-13$ 
[2] Hafez, M.M., Guo, W.H., Nonuniqueness of transonic flows, Acta Mechanica, 1999, 138, no. 3-4, 177-184

[3] Kuzmin, A., Aerodynamic surfaces admitting jumps of the lift coefficient in transonic flight, in: Hafez M.M., Oshima K., Kwak D. (Eds), Comput Fluid Dynamics Review 2010, World Scientific, Singapure, 2010

[4] Kuzmin, A., Non-unique transonic flows over airfoils, Computers and Fluids, 63, 2012, 1-8

[5] Jameson, A., Vassberg, J.C., Ou, K., Further studies of airfoils supporting non-unique solutions in transonic flow, AIAA Paper, 2011, 2011-3509, 1-22

[6] Kuzmin, A., Adverse free-stream conditions for transonic airfoils with concave arcs, In: Kuzmin A. (Ed), Comput Fluid Dynamics 2010, Springer, 2011, 887891

[7] Zores R., Transonic airfoil design with expert systems, AIAA Paper, 1995, 95-1818, 1-8

[8] Lane, K.A., Marshall, D.D., A surface parametrization method for airfoil optimization and high lift 2D geometries utilizing the CST methodology, AIAA Paper, 2009, 2009-1461, 1-13

[9] Milholen W.E., Owens L.R., On the application of contour bimps for transonic drag reduction, AIAA Paper, 2005, 2005-0462, 1-19

[10] Lee, D.S., Periaux, J., Srinivas, K., Gonzalez, L.F., Qin, N., Onate, E., Shock control bump design optimization on natural laminar aerofoil, In: Kuzmin A. (Ed), Comput Fluid Dynamics 2010, Springer, 2011, 253-
259

[11] Padovan, L., Pediroda, V., Poloni, C., Multiobjective robust design optimization of airfoils in transonic field, Multidisciplinary Methods for Analysis Optimization and Control of Complex Systems, Mathematics in Industry, 2005, 6, 283-295

[12] Huyse, L., Lewis, R.M., Aerodynamic shape optimization of two-dimensional airfoils under uncertain conditions, NASA/CR-2001-210648, ICASE Report, 2001

[13] Barth T.J., Jespersen, D.C., The design and application of upwind schemes on unstructured meshes, AIAA Paper, 1989, 89-0366

[14] ANSYS CFX-11.0 user manual. ANSYS Inc., Canonsburg, USA, 2006

[15] Merchant, A.A., Design and analysis of supercritical airfoils with boundary layer suction, Master's thesis, Massachusetts Instit. Technology, 1996

[16] Collyer, M.R. An extension to the method of Garabedian and Korn for the calculation of transonic flow past an airfoil to include the effects of a boundary layer and wake, Aeronautical Research Council, Reports and Memoranda, RAE Farnborough, Hants, 3828, 1978

[17] Moelyadi, M.A., Improvement of transonic aerofoil aerodynamic performance with trailing edge modification using wedge configuration, ICAS Paper, 2002, 2002-R44 